\title{
A CLINICAL TRIAL TO EVALUATE THE SAFETY AND EFFICACY OF SAROGLITAZAR COMPARED TO FENOFIBRATE IN PATIENTS WITH DYSLIPIDEMIA
}

\author{
D. Parmar ${ }^{1}$, M. Krishnappa ${ }^{2}$, F. Arifahmed ${ }^{1}$, N. Mali², J. Patel², M. Shah², K. Parmar², C. \\ $\mathrm{Shah}^{2}$, K. Faldu² \\ 1Zydus Discovery DMCC, Clinical R\&D, Dubai, United Arab Emirates \\ 2Zydus Research Centre-Cadila Healthcare Ltd, Clinical R\&D, Ahmedabad, India
}

\begin{abstract}
Background and Aims
Dyslipidemia is a strong predictor for cardiovascular diseases and its prevalence is rising rapidly worldwide. Saroglitazar (Lipaglyn ${ }^{\circledR}$; Zydus Cadila, Ahmedabad, India), a novel dual peroxisome proliferator activated receptor agonist, is approved in India for management of diabetic dyslipidemia and hypertriglyceridemia in type 2 diabetes mellitus not controlled by statin alone. To evaluate the effect of saroglitazar in dyslipidemia, this 24-week clinical trial was conducted from December-2014 to October-2017 in India.
\end{abstract}

\section{Methods}

This was a multicentre, randomized, double-blind study conducted to evaluate the safety and efficacy of saroglitazar 2 $\mathrm{mg}$ and $4 \mathrm{mg}$ compared to fenofibrate $160 \mathrm{mg}$ in adult patients with hypertriglyceridemia ( $\geq 200 \mathrm{mg} / \mathrm{dL}$ ). Total 997 subjects at 35 centres were randomized in a ratio of 2:2:1 to saroglitazar $2 \mathrm{mg}$, $4 \mathrm{mg}$, and fenofibrate $160 \mathrm{mg}$, respectively. Efficacy evaluation of triglyceride (TG) (primary outcome) and other lipid parameters was conducted at week-12 and week-24 from baseline.
Results

Saroglitazar $2 \mathrm{mg}$ and $4 \mathrm{mg}$ significantly reduced TG at week-12 and week-24 from baseline (Table-1). Saroglitazar $2 \mathrm{mg}$ and 4 $\mathrm{mg}$ significantly reduced total cholesterol, very low-density lipoprotein, non high-density lipoprotein (HDL), apolipoprotein B and significantly increased HDL at week-12 and week-24 from baseline (Table-1). Results for the per-protocol and modified intention-to-treat populations were similar. No serious adverse events or deaths reported in the study groups.

\section{Conclusions}

Saroglitazar was found to be efficacious and safe in patients with dyslipidemia.

Table-1: Change in efficacy outcomes from baseline at week-12 and week-24 in the per-protocol population

\begin{tabular}{|c|c|c|c|c|}
\hline Efficacy Outcomes & Assessment at Study Time Point & $\begin{array}{l}\text { Saroglitazar } \\
2 \mathrm{mg}(\mathrm{n}=134) \\
\text { Mean } \pm \mathrm{SD}\end{array}$ & $\begin{array}{l}\text { Saroglitazar } \\
4 \mathrm{mg}(\mathrm{n}=146) \\
\text { Mean } \pm \mathrm{SD}\end{array}$ & $\begin{array}{l}\text { Fenofibrate } \\
160 \mathrm{mg}(\mathrm{n}=67) \\
\text { Mean } \pm \mathrm{SD}\end{array}$ \\
\hline \multirow[t]{3}{*}{$\mathrm{TG}(\mathrm{mg} / \mathrm{dL})$} & Baseline & $360.36 \pm 163.60$ & $345.32 \pm 153.88$ & $345.42 \pm 134.39$ \\
\hline & Absolute change at week-12 & $-201.74 \pm 173.18^{*}$ & $-181.56 \pm 168.19^{*}$ & $-174.31 \pm 151.67^{\star}$ \\
\hline & Absolute change at week-24 & $-201.10 \pm 181.39^{*}$ & $-182.96 \pm 168.42^{*}$ & $-201.67 \pm 134.04^{*}$ \\
\hline \multirow[t]{3}{*}{$\mathrm{LDL}(\mathrm{mg} / \mathrm{dL})$} & Baseline & $127.81 \pm 44.55$ & $124.75 \pm 43.83$ & $128.23 \pm 39.83$ \\
\hline & Absolute change at week-12 & $-9.23 \pm 54.70$ & $-6.14 \pm 49.38$ & $-9.73 \pm 52.53$ \\
\hline & Absolute change at week-24 & $-18.22 \pm 60.32^{*}$ & $-10.26 \pm 54.83^{*}$ & $-19.63 \pm 49.44^{*}$ \\
\hline \multirow[t]{3}{*}{ VLDL (mg/dL) } & Baseline & $72.07 \pm 32.72$ & $68.92 \pm 30.89$ & $68.72 \pm 27.17$ \\
\hline & Absolute change at week-12 & $-40.35 \pm 34.64^{\star}$ & $-36.17 \pm 33.75^{\star}$ & $-34.50 \pm 30.38^{*}$ \\
\hline & Absolute change at week-24 & $-40.22 \pm 36.28^{*}$ & $-36.45 \pm 33.77^{\star}$ & $-39.97 \pm 26.98^{*}$ \\
\hline \multirow[t]{3}{*}{$\mathrm{HDL}(\mathrm{mg} / \mathrm{dL})$} & Baseline & $36.90 \pm 9.12$ & $37.60 \pm 11.91$ & $39.75 \pm 11.77$ \\
\hline & Absolute change at week-12 & $6.28 \pm 12.97^{*}$ & $5.55 \pm 12.55^{*}$ & $5.03 \pm 12.62^{*}$ \\
\hline & Absolute change at week-24 & $6.00 \pm 14.50^{*}$ & $5.76 \pm 15.54^{*}$ & $6.54 \pm 16.59^{\star}$ \\
\hline \multirow[t]{3}{*}{ Total cholesterol (mg/dL) } & Baseline & $205.19 \pm 44.84$ & $198.11 \pm 43.27$ & $204.82 \pm 42.80$ \\
\hline & Absolute change at week-12 & $-27.66 \pm 55.74^{*}$ & $-22.46 \pm 51.76^{*}$ & $-25.34 \pm 52.47^{*}$ \\
\hline & Absolute change at week-24 & $-32.36 \pm 61.34^{*}$ & $-21.97 \pm 58.58^{\star}$ & $-32.21 \pm 53.29^{*}$ \\
\hline \multirow{3}{*}{ Non-HDL (mg/dL) } & Baseline & $168.28 \pm 44.24$ & $160.53 \pm 40.38$ & $165.07 \pm 42.07$ \\
\hline & Absolute change at week-12 & $-33.92 \pm 54.86^{*}$ & $-28.01 \pm 51.32^{*}$ & $-30.36 \pm 53.54^{*}$ \\
\hline & Absolute change at week-24 & $-38.33 \pm 60.56^{*}$ & $-27.77 \pm 54.88^{*}$ & $-38.70 \pm 50.49^{*}$ \\
\hline \multirow[t]{3}{*}{ Apo A1 (mg/dL) } & Baseline & $130.94 \pm 33.56$ & $123.71 \pm 30.64$ & $130.46 \pm 33.97$ \\
\hline & Absolute change at week-12 & $-3.36 \pm 38.14$ & $0.88 \pm 36.09$ & $0.08 \pm 48.51$ \\
\hline & Absolute change at week-24 & $-7.20 \pm 38.04^{*}$ & $-0.54 \pm 39.69$ & $-2.37 \pm 41.35$ \\
\hline \multirow[t]{3}{*}{ Apo B (mg/dL) } & Baseline & $122.48 \pm 32.78$ & $123.71 \pm 30.64$ & $118.94 \pm 35.03$ \\
\hline & Absolute change at week-12 & $-22.57 \pm 41.78^{*}$ & $-16.33 \pm 36.14^{*}$ & $-16.31 \pm 44.26^{*}$ \\
\hline & Absolute change at week- 24 & $-21.24 \pm 44.85^{\star}$ & $-15.26 \pm 37.07^{*}$ & $-21.72 \pm 38.75^{\star}$ \\
\hline \multicolumn{5}{|c|}{$\begin{array}{l}\text { *Significant difference compared to baseline using paired t-test; } \\
\text { Absolute change at week-12 = value at week- } 12-\text { value at baseline } \\
\text { Absolute change at week- } 24=\text { value at week- } 24-\text { value at baseline }\end{array}$} \\
\hline \multicolumn{5}{|c|}{$\begin{array}{l}\text { Abbreviations: SD, standard deviation; TG, triglyceride; LDL, low-density lipoprotein; VLDL, very low-density lipoprotein; HDL, high- } \\
\text { density lipoprotein; Apo, apolipoprotein; mg, milligram; dL, deciliter }\end{array}$} \\
\hline
\end{tabular}

